\title{
Acute Pancreatitis and Pregnancy
}

\author{
Bensal Abdelhak, Belhamidi Said $_{1}$, Moutaoukil Mohamed 2 \\ Bouchentouf Sidi Mohamed $_{1}$, Moujahid Mountacer 1 , Bounaim Ahmed \\ ${ }_{1}$ Department of Visceral Surgery -I- MVMIH - Rabat-Morroco \\ ${ }_{2}$ Departement of Reanimation-Anesthesia
}

\begin{abstract}
Acute pancreatitis is a rare condition during pregnancy. Gallstones are the most common etiology.Its diagnosis should not suffer from delay because its evolution can jeopardize the vital maternal and fetal prognosis.We report the observation of woman, 27 years old, pregnant at 27 weeks of amenorrhea. She was admitted for epigastric pain evolving for four days, her lipasemia was at $\mathbf{3 6 0}$ IU / L. Abdominopelvic ultrasound showed a thin-walled lithiasic gallbladder, the non-dilated intra and extra hepatic bile ducts and an enlarged pancreas; Active pregnancy with presence of fetal heart activity. MRI performed immediately objectified a multilithiasic gallbladder with moderate dilation of the common bile duct, which is the site of 3 non-obstructive microlithiasis, the pancreas is enlarged. The patient was put on rest with a diet low in lipids, good rehydration (basic ration with electrolyte supplementation, symptomatic treatment. satisfactory check-up and she left after five days of hospitalization with a check-up and possible cholecystectomy after childbirth.
\end{abstract}

Acute pancreatitis rarely occurs during pregnancy; the reported frequency ranges from 1 in 1000 to 3 in 10000 births. The most common etiology is cholelithiasis in $70 \%$ of cases. According to Pitchumoni, more than $50 \%$ of acute pancreatitis occurs, as in our patient, in the 3rd trimester. The clinical symptomatology and a high lipasemia allow us to make the diagnosis of acute pancreatitis. The abdominal ultrasound confirms the biliary etiology, however she does not appreciate the pancreas. The abdominal scanner cannot be performed due to the risk of irradiation, we performed a biliMRI whose sensitivity is $90 \%$ without risk either for the mother or for the fetus. The management of acute benign pancreatitis is done in collaboration with gynecologists and is based on the diet to put to rest the endocrine function of the pancreas and symptomatic treatment with a good prognosis. In severe forms or associated with cholangitis management is multidisciplinary with a prognosis reserved for the mother. A delay in diagnosis and treatment worsens the prognosis. The medical treatment is identical to acute pancreatitis outside of pregnancy, however there remains the specific treatment of acute pancreatitis secondary to cholelithiasis, because on the one hand we must consider the high rate of recurrences during pregnancy estimated at $70 \%$, and on the other hand consider both the maternal risk which depends on the anatomical type of acute pancreatitis and the gestational age of onset, and the fetal risk which depends on the severity of the clinical form.

Keywords:- Acute Pancreatitis, Pregnancy and abdominal pain.

\section{INTRODUCTION}

Acute pancreatitis is a rare condition during pregnancy $[1,2]$. Gallstones are the most common etiology $[1,2]$. It occurs in most cases during the third trimester of pregnancy $[2,3]$. Its diagnosis should not suffer from delay because its evolution can jeopardize the vital maternal and fetal prognosis.

\section{MATRIELS AND METHODS}

We report the observation of Mrs. B.S, 27 years old, married, 3 gestures and 2 parities, carrying a doubly scarred uterus, pregnant at 27 weeks of amenorrhea and having no particular pathological history. She was admitted to the medical and surgical emergency department for epigastric pain with types of cramps radiating to the back and bilious vomiting, evolving for four days. The clinical examination revealed a patient with a good general condition, nonpyretic, blood pressure at $11 / 07 \mathrm{mmHg}$, with a tenderness of the right hypochondrium on the abdominal examination. The gynecological examination shows the presence of greenish leukorrhea and vulvar pruritus. The biological assessment showed a leukocytosis at 14.8.10 (3) / mm (3) predominantly polynuclear neutrophils (84\%), a CRP at $288 \mathrm{mg} / \mathrm{1}$, cytolysis with ASAT at $52 \mathrm{IU} / \mathrm{L}$ and ALT at $56 \mathrm{IU} / \mathrm{L}$, a biological cholestasis with GGT at $125 \mathrm{IU} / \mathrm{L}$ and PAL at $146 \mathrm{IU} / \mathrm{L}$ and a lipasemia at $360 \mathrm{IU} / \mathrm{L}$. Abdominopelvic ultrasound showed a thin-walled lithiasic gallbladder, the non-dilated intra and extra hepatic bile ducts and an enlarged pancreas; Active pregnancy with presence of fetal heart activity. MRI performed immediately objectified a multilithiasic gallbladder with moderate dilation of the common bile duct, which is the site of 3 non-obstructive microlithiasis, the pancreas is enlarged [fig.1]. The patient was put on rest with a diet low in lipids, good rehydration (basic ration with electrolyte supplementation, symptomatic treatment: analgesic, antispasmodic and antiemetic, gastric protector (PPI) and rigorous clinical monitoring. has progressed well with a satisfactory check-up and she left after five days of hospitalization with a check-up and possible cholecystectomy after childbirth. 


\section{RESULTS}

Acute pancreatitis rarely occurs during pregnancy; the reported frequency ranges from 1 in 1000 to 3 in 10000 births [1]. The most common etiology is cholelithiasis in $70 \%$ of cases [1,2]. According to Pitchumoni [1], more than $50 \%$ of acute pancreatitis occurs, as in our patient, in the 3rd trimester. During the latter, the pregnant uterus can compress the common bile duct and cause acute pancreatitis [6]. The clinical symptomatology and a high lipasemia allow us to make the diagnosis of acute pancreatitis [8], in our patient it was more than three times normal,this dosage should not be subject to any hesitation in order to reach an early diagnosis. The abdominal ultrasound confirms the biliary etiology and appreciates the presence or not of a dilation of the bile ducts [7], we were able to do it in our patient, however she does not appreciate the pancreas. The abdominal scanner cannot be performed due to the risk of irradiation, we performed a biliMRI whose sensitivity is $90 \%$ without risk either for the mother or for the fetus [3]. The management of acute benign pancreatitis is done in collaboration with gynecologists and is based on the diet to put to rest the endocrine function of the pancreas and symptomatic treatment with a good prognosis $[1,4,5]$, attitude that we have adopted for our patient. In severe forms or associated with cholangitis management is multidisciplinary, antibiotic therapy is undertaken by avoiding molecules that may be teratogenic and by adjusting the doses, with a prognosis reserved for the mother [2]. A delay in diagnosis and treatment worsens the prognosis. We must think of acute pancreatitis in front of any abdominal pain in a pregnant woman. Thus, Gangat found that $30.76 \%$ of maternal mortality in case of acute pancreatitis in pregnant women is linked to the diagnostic and therapeutic delay [9].Our patient was diagnosed early enough, hence her good progress and discharge after only five days of hospitalization. The medical treatment is identical to acute pancreatitis outside of pregnancy, however there remains the specific treatment of acute pancreatitis secondary to cholelithiasis, because on the one hand we must consider the high rate of recurrences during pregnancy estimated at $70 \%$ versus $30 \%$ in the general population, and on the other hand consider both the maternal risk which depends on the anatomical type of acute pancreatitis and the gestational age of onset, and the fetal risk which depends on the severity of the clinical form, this risk is represented by abortion, acute fetal distress and fetal death in utero which is estimated at $20 \%$ [10]. The benefit of cholecystectomy has been demonstrated by the absence of maternal or fetal intraoperative complications. In a study of 85 cases: 24 cholecystectomy, all performed under laparotomy, 5 in the first trimester, 11 in the second and 8 postpartum and 61 other patients $(85 \%)$ had been treated medically, without maternal or fetal mortality. However, a new outbreak of acute pancreatitis occurred in $69 \%$ of the cases. These recurrences required 5 emergency interventions [1]. the treatment must be adapted on a case-by-case basis according to the gestational age: first trimester: therapeutic abstention and cholecystectomy in the second trimester; Second trimester: laparoscopic cholecystectomy because the risk of miscarriage is halved [11] similarly, the rate of premature birth is zero, while it is evaluated at $40 \%$ when the intervention takes place in the third trimester ; Third trimester: sphincterotomy; Postpartum cholecystectomy is essential [12]. Our patient presented with a benign acute pancreatitis, she progressed well under medical treatment,we opted for a cholecystectomy after childbirth.

\section{CONCLUSION}

Acute pancreatitis is rare during pregnancy; we have to think about it in front of any abdominal pain in a pregnant woman. The clinic and the elevation of lipasemia helped by ultrasound and biliRMI allow to make a positive diagnosis, which must not suffer any delay; and possibly etiological. Management is often multidisciplinary and must be adapted on a case-by-case basis.

\section{REFERENCES}

[1]. Pitchumoni Capecomorin S, yegneswaran balaji.acute pancrestitis in pregnancy.World J Gastroenterol. 2009; 15 (45): 5641-5646.

[2]. Ducarme G, Maire F, Chatel p, Luton D, Hammel P. Acute pancreatitis during preganacy: a review.J Perinatol. 2014; 34 (2): 87-94.

[3]. Severe acute pancreatitis on pregnancy in black Africans: about a case: I. Lawani, ARkpossou, B. Noukpozounkou, FHRgnangnon, YISouaibou, DGDansougbessi, B. Hounkpation, FMDossou, J. Olory- Togbe.The Pan African Medical Journal. 2017; 26: 175.

[4]. Pancreatitis and Pregnancy: J. Medarhri, B. lekhal, M.elouanani, A.Ikken, M.cherrab, M.Amraoui, A.Errougani, A.Benchekroun, R.Chkoff, S.Balafrej. Maghreb Medicine $1997 \mathrm{n}^{\circ} 63$.

[5]. Pancreation aiue and Pregnancy: D. Karsenti, Y. Bacq, La Lettre Du L'hépato-Gastroloue-N ${ }^{\circ}$ 4-vol.VJuillet-Aout2002.

[6]. T.Shou-Jiang, R.Edmundo, S.Sundeep, M.MarlynJ, J.SaadF, S.Jayaprakash, L.Luis F, R.Doon C.Acute pancreatitis durring pregnancy.Clin Gastroenterol hepatol.2010; 8 (1): 85-90.

[7]. Stimac Davor, Stimac Tea.acute pancreatitis during pregnancy.Eur J Gastroenterol Hepatol.2011; 23 (10): 839-844.

[8]. High health authority.Assessment of Amylasemia and Lipasemia for the initial diagnosis of acute pancreatitis. Bio Trib Mag. 2009; 32 (1): 29-31.

[9]. G.Shoaib, R.Anjum, A.Mammammad, F. Saber, A.AmeerM.I.Ahmed.Freqency of Acute pacreatiis in pregnancy Andit's Outcome.Pakistan J Surg. 2009; 25 (2): 69-71.

[10]. Jayi S, Bouguern H, Chaara NBanani A, MelhoufMA. About a pseudo-occlusive syndrome following a caesarean. Medical Hope. 2008 December; 15: No. 153. 
ISSN No:-2456-2165

[11]. Jamidar PA, Beck GJ, hoffman BJ, et al. Endoscopic retrograde cholangiopancretography in pregnancy. am Gastroenterol. 1995; 90 (8): 1263-7.
[12]. S.Amourak, S.Jayi, F.F.Aloui, h.Bouguern, H.Chaara, M.A.Melhouf.PanAfrMedJ.2015; 20: 185

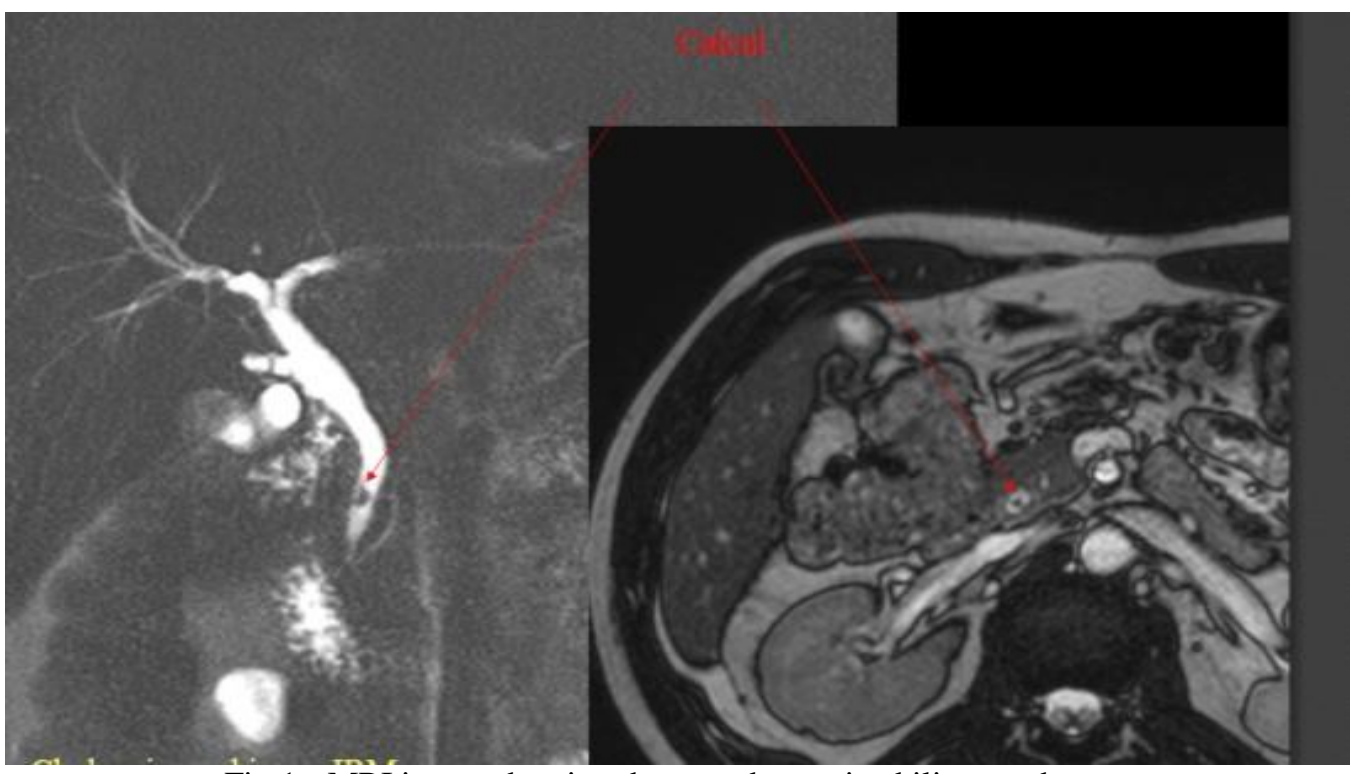

Fig 1:- MRI image showing the non obstructive bilious pathways 